\title{
DESARROLLO DE ADOQUINES A PARTIR DE LOS RELAVES DE MINA.
}

\section{Development of pavers from mine tailings.}

Jácome Calderón Juan Francisco* iD, Burbano Morillo Danny Santiago iD, Núñez Romero Jorge Alberto iD.

Universidad Central del Ecuador, Facultad de Ingeniería en Geología, Minas, Petróleos y Ambiental, Quito, Ecuador.

*juancho_jacome@hotmail.es

$\mathrm{R}$ esumen

El constante desarrollo minero ha generado una gran cantidad de pasivos ambientales, especialmente relaves mineros (residuos mineros), almacenados en relaveras, la mayoría de los cuales son abandonados después de su vida útil, sin mantener un control químico y físico, generando contaminación ambiental. El desarrollo de adoquines empleando los relaves de mina empieza con los análisis: físicos, mineralógicos y químicos de las arenas de relave, para determinar si el material es apto como agregado. Una vez analizado el relave, se realizó prototipos de adoquines usando diferentes dosificaciones reemplazando el polvo de piedra con el relave y así determinar las mejores características de resistencia física apegados a la normativa INEN 3040 e INEN 1488 1986. Se determinó que la dosificación adecuada como agregado de relave es reemplazando en un $70 \%$ al polvo de piedra en la fabricación de adoquines. Se concluye que mortero diseñado en base a arena de relave, cemento y agregados representa un ahorro al momento de gestionar estos residuos en relación a la construcción de nuevas relaveras. Se obtuvo una resistencia a la tracción de $3 \mathrm{MPa}$, cuyo valor se encuentra ligado al alto contenido de cuarzo $( \pm 80 \%)$, propio de la paragénesis mineral del yacimiento.

Palabras clave: Relave, adoquín, resistencia, pasivo ambiental, drenaje ácido.

A bstract

The constant mining development has generated a large number of environmental liabilities, especially mine tailings (mining waste), stored in tailings, most of which are abandoned after their useful life, without maintaining chemical and physical control, generating environmental contamination. The development of pavers using mine tailings begins with the physical, mineralogical and chemical analyzes of the tailings sand, to determine if the material is suitable as an aggregate. Once the tailings were analyzed, prototypes of pavers were made using different dosages, replacing the stone powder with the tailings in order to determine the best physical resistance characteristics according to INEN 3040 and INEN 14881986 standards. It was determined that the adequate dosage as tailings aggregate is replacing $70 \%$ of the stone dust in the manufacture of pavers. It is concluded that mortar designed based on tailings sand, cement and aggregates represents a saving at the time of managing these residues in relation to the construction of new tailings. A tensile strength of $3 \mathrm{MPa}$ was obtained, whose value is linked to the high quartz content $( \pm 80 \%)$, typical of the mineral paragenesis of the deposit.

Keywords: Tailings, paver, resistance, environmental liability, acid drainage.

Fecha de recepción: 05-04-2021

\section{INTRODUCCIÓN}

La explotación minera en Sudamérica se ha realizado ya por los Incas desde el tiempo Pre-Colombino, pero los datos muestran que desde el año 1556 se ha encontrado la primera contaminación causada por la explotación minera, principalmente por el uso de agua y el depósito de sus desechos (1). A partir del año 1800 la tecnificación del procesamiento de minerales en 
el mundo causo que las partículas remanentes y relaves empezaron a ser acumulados en sectores convenientes para el sector minero de ese tiempo, principalmente en lugares cercanos a arroyos y ríos para que el agua arrastre el material (2). De esta manera para el año 1900 inició con el florecimiento de más distritos mineros se aumentó gradualmente la descarga descontrolada de relaves y la construcción de las primeras piscinas para almacenar relaves $(3,4)$.

Entre los principales desafíos globales críticos que pueden poner en riesgo a las presentes y futuras generaciones, se encuentran: la degradación de ecosistemas, calentamiento global y agotamiento de recursos naturales $(5,6)$. Es por ello que la gestión ambientalmente racional de los desechos, entre estos los relaves, siendo los residuos finos y agua resultantes de procesos de lixiviación y concentración de minerales debe ser considerada una de las principales preocupaciones para mantener la calidad del ambiente y de la vida humana en la actualidad (7).

Es por eso que la investigación de uso de los relaves como material para fabricar elementos de construcción, busca una solución al problema de la mayoría de empresas mineras del país, por la falta de espacios para depositar los residuos del procesamiento mineral (8).

Falta de Investigación sobre el uso o aprovechamiento de los relaves de la mina "Agro-Industrial El Corazón-Ecuador”, a partir de ensayos de laboratorio, limitan la toma de decisiones sobre su utilización en varios sectores de la industria como por ejemplo en la construcción y obras civiles (9). Además, la generación diaria de relaves producto del procesamiento mineral, deriva en una constante búsqueda de soluciones óptimas para la gestión y almacenamiento de estos residuos.

Al buscar nuevas alternativas para los relaves, que no sea su confinamiento y abandono, la minería obliga a tomar decisiones que sean amigables en el entorno socio-ambiental desde la extracción mineral hasta la recuperación del metal valioso (10), de esta manera el beneficio iría más allá de la rentabilidad económica para la empresa y se llegaría a un apoyo social con nuevas ideas y estrategias enfocadas a la gestión de los residuos mineros (11).

De esta forma, en base a una caracterización física, química y mineralógica de los relaves de mina se promueve la investigación y estudio de la factibilidad de usar estos desechos mineros como agregados en la fabricación de adoquines y así buscar una solución a la falta de áreas para la construcción de nuevas relaveras (12). Un factor determínate que condiciona el aprovechamiento de los relaves en la fabricación de los adoquines, es la resistencia física, donde la dosificación adecuada permite obtener un producto acorde a la normativa ecuatoriana INEN $30402016(13,14)$. La fase de experimentación incluye 48 muestras de relaves (proceso de cianuración) obtenidos de la empresa Agroindustrial El Corazón, mina que se ubica al Nor-Occidente de la ciudad de Quito-Ecuador (Figura 1); de las cuales se obtuvo 60 adoquines divididos en 3 tipos de dosificaciones, que fueron ensayados hasta obtener el adoquín que cumple con los lineamientos de la normativa INEN 3040 e INEN 14881986 (15).

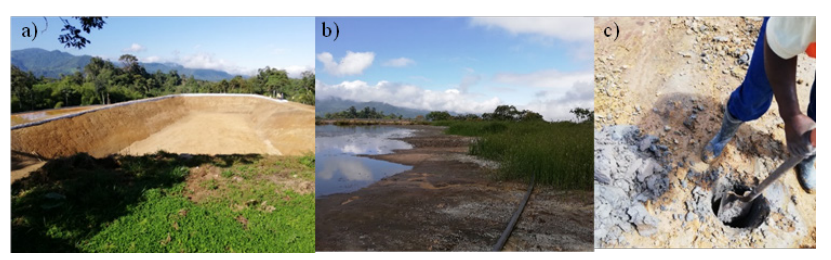

Figura 1. a) Vista panorámica de la construcción de las relaveras en Agroindustrial El Corazón b) Estado actual de la relavera \#9 c) Malla de muestreo de Relaves.

\section{MATERIALES Y MÉTODOS}

El proceso de investigación se basa en el análisis de las características físicos-químicas y mineralógicas de la materia prima (relaves) y del producto resultante (adoquines).

En la Figura 2 se detalla el proceso metodológico iniciando por; topografía de la relavera, insumo que permite el cálculo de volumen, malla de muestreo, técnica empleada para obtener las arenas de relave las cuales pasaron por un tratamiento de homogeneización y cuarteo hasta obtener muestras selectivas que posteriormente pasaron por un análisis de laboratorio determinando así: la granulometría, peso específico, hu- 
medad, Test ABA y difracción de rayos X.

El Test ABA es de mucha importancia en el ámbito minero debido que el resultado de los mismos es una predicción sobre la posibilidad de que genere drenaje ácido una roca o una mina, a partir de resultados de laboratorio que determina el porcentaje de contenido de sulfatos o sulfuros; permitiendo planificar el control ambiental en la industria minera $(16,17)$. El ensayo básicamente consiste en la posibilidad de generar drenaje acido del material en relación a la posibilidad del mismo material de neutralizar el posible drenaje ácido.

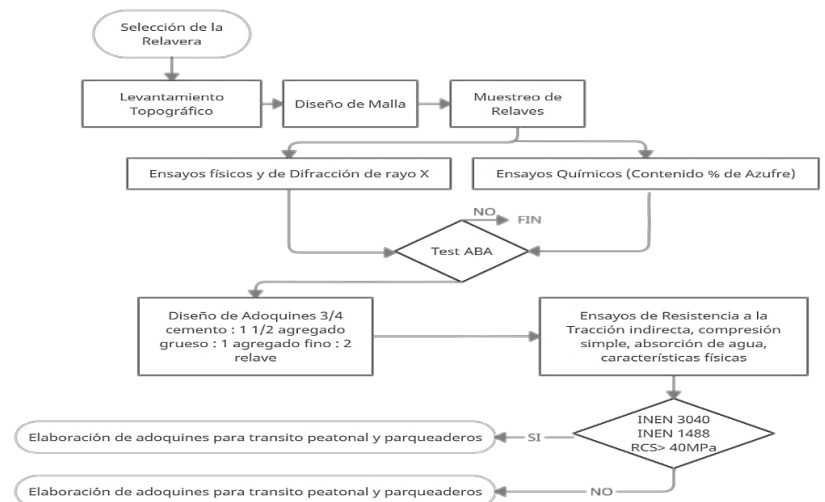

Figura 2. Flujo grama de muestreo y Ensayos a relaves

Los resultados favorables obtenidos de laboratorio en relación a sus propiedades físico (granulometría) y químicas (menor presencia de sulfuros) permitieron tomar decisiones para el empleo de los relaves en la fabricación de adoquines tipo centauro.

Los adoquines para cumplir la resistencia requerida fueron fabricados en base a una dosificación calculada de la mezcla de cemento, agua, agregado grueso y agregado fino (polvo de piedra). La relación de agregados que intervienen en la fabricación del adoquín se condiciona al uso de agregado fino o polvo de piedra con las siguientes 3 variaciones: reemplazando en un 30\%, un 50\% y un $70 \%$ de la cantidad agregado fino por relave. La dosificación que presente mejores propiedades físicas será la utilizada para la fabricación del producto en base a la normativa INEN 3040 del año 2016.

En la tabla 1 se especifica la dosificación en volumen utilizada en la fabricación de los diferentes prototipos.

\begin{tabular}{clll}
\hline & $\begin{array}{l}\text { Mezcla 1 } \\
\text { (relave 30\%) }\end{array}$ & $\begin{array}{l}\text { Mezcla 2 } \\
\text { (relave 50\%) }\end{array}$ & $\begin{array}{l}\text { Mezcla 3 } \\
\text { (relave 70\%) }\end{array}$ \\
\hline Cemento & $3 / 4$ & $3 / 4$ & $3 / 4$ \\
\hline Agregado grueso & $1 \frac{1}{2}$ & $1 \frac{1}{2}$ & $1 \frac{1}{2}$ \\
\hline Agregado fino & 2 & $1 \frac{1}{2}$ & 1 \\
\hline Relave & 1 & $1 \frac{1}{2}$ & 2 \\
\hline
\end{tabular}

Tabla 1. Dosificación utilizada en los diferentes prototipos.

Los adoquines tipo centauro fabricados bajo las relaciones anteriormente citadas cumplieron con los ensayos enmarcados en la normativa INEN 3040 2016; que incluye ensayos de tracción indirecta, absorción total de agua (permeabilidad) y características físicas.

Por otro lado, Yugsi, (18) menciona que, a partir de los resultados de la tracción indirecta medida en los adoquines, se puede obtener la resistencia a la compresión mediante la ecuación de Adam M. Nevill (1).

$$
\mathrm{fc}=(\mathrm{ft} / 0.3)^{(3 / 2)}
$$

Donde:

$\mathrm{ft}=$ Resistencia a la tracción indirecta. $\mathrm{fc}_{\mathrm{c}}=$ Resistencia a la compresión simple.

\section{RESULTADOS Y DISCUSIÓN}

\section{Análisis del Relave}

En la tabla 2 se detalla promedio de ensayos de laboratorio; mineralógico, granulométrico e índices, obtenidos de un total de 48 muestras de relave tomados dentro de una configuración de una malla de $5 \mathrm{~m} \times 5 \mathrm{~m}$, alcanzando una profundidad promedio de $1.5 \mathrm{~m}$.

\begin{tabular}{ccll}
\hline \multicolumn{2}{c}{ Descripción } & Cantidad & Medida \\
\hline MINERALOGÍA & Cuarzo & 78.8 & $\%$ \\
& Caolinita & 10.6 & $\%$ \\
& Plagioclasas & 4.2 & $\%$ \\
& Moscovita & 3.8 & $\%$ \\
& Hematita & 1.6 & $\%$ \\
& Anatasa & 1 & $\%$ \\
\hline GRANULOMETRÍA & Arena Fina & 41.6 & $\%$ \\
& Limo - Arcilla & 58.4 & $\%$ \\
\hline \multicolumn{2}{c}{ Peso Específico } & 18.82 & $\%$ \\
\hline
\end{tabular}

Tabla 2. Resumen de propiedades mineralógicas y físicas.

\section{Test ABA}

Las muestras representativas de los relaves fueron enviadas al Laboratorio GRUNTEC de don- 
de los resultados se reflejan en la tabla 3.

\begin{tabular}{|c|c|}
\hline Especies de Azufre & \\
\hline Azufre total en Sulfato \% & 0.13 \\
\hline Potencial de Neutralización por Carbonato $\mathrm{kgCaCO}_{3} / \mathrm{t}$ & 4.7 \\
\hline
\end{tabular}

Aplicando la ecuación (2) para determinar la posible generación de drenaje ácido por parte de los relaves y por ende de los productos que se espera obtener (19). Los resultados son los siguientes:

$31.25 \times \mathrm{S} \%=\mathrm{APP}(2)$

$31.25 \times 0.13=4.063$

Dando como resultado los siguientes datos.

APP $($ acid production potential $)=4.063$

$\mathrm{NP}($ neutralization potential $)=4.7$

A partir de los resultados obtenidos de APP y NP, se calcula dos medidas del TEST ABA los cuales son PNN y la relación N/APP $(3,4)$.

$\mathrm{PNN}=\mathrm{NP}-\mathrm{APP}(3)$

$\mathrm{PNN}=4.7-4.063$

$\mathrm{PNN}=0.64$

$\mathrm{NP} / \mathrm{APP}=4.7 / 4.063=1.16(4)$

Los valores obtenidos se interpretan con la tabla 4 del Test ABA.

\begin{tabular}{|c|c|c|c|}
\hline & PNN & 'PN/PA & INTERPRETACIÓN \\
\hline & $>20$ & $>3$ & $\begin{array}{l}\text { Bajo o Nulo potencial de } \\
\text { Generación de ácido }\end{array}$ \\
\hline & $<20$ & 1 a 3 & $\begin{array}{l}{ }^{*} \text { Potencial Marginal de } \\
\text { generación de ácido }\end{array}$ \\
\hline & Negativo & $<1$ & $\begin{array}{l}\text { Alto Potencial de generación } \\
\text { de ácido }\end{array}$ \\
\hline \multirow[t]{2}{*}{-20 y 20} & \multirow{2}{*}{$\begin{array}{l}\text { Interpretación } \\
\text { dificultosa }\end{array}$} & $>3$ & $\begin{array}{l}\text { Bajo riesgo de formar drena- } \\
\text { je ácido }\end{array}$ \\
\hline & & Entre 1 y 3 & Zona de incetidumbre \\
\hline
\end{tabular}

Tabla 4. Interpretación TEST ABA

Nota: $\left.{ }^{*}\right)$ resultado de Test ABA

Fuente: U.S Environmental Protection Agency, 1994

El porcentaje de minerales presentes en el relave se determinó mediante en el ensayo de difracción de Rayos X, donde se observa que el cuarzo y la caolinita predominan en el material, evidenciando la alteración argílica insipiente del yacimiento $(20,21,22)$, esto crea la posibilidad de utilización del relave al no generar un drenaje ácido, sumado a la poca o nula presencia de óxidos, sulfuros y sulfatos (23).

Sumado a lo anteriormente expuesto, el Test
ABA dio como resultado un "potencial marginal" de generar drenaje ácido por parte de los relaves, proporcionando una mayor seguridad en la toma de la decisión para el uso de los relaves que fueron estudiados. Con este resultado se presume que al aumentar más carbonato al relave (cemento) permite una efectiva inertización de las arenas de relave, en consecuencia, el adoquín diseñado no provocará un pasivo ambiental (24). En base a los resultados químicos favorables del uso de relaves, se procedió a elaborar 3 prototipos de adoquines con diferentes dosificaciones (cemento + agregado grueso + agregado fino + relave) donde la resistencia a la tracción indirecta es el parámetro guía para la selección de la opción más adecuada (25).

En la tabla 5 se indica las principales características físicas de los 3 diferentes prototipos de adoquines, es importante mencionar que el número de ensayos dependió de los requeridos por la normativa actual INEN 3040 (2016).

\begin{tabular}{|c|c|c|c|c|}
\hline Prototipo & \% de Relave & Tipo de Ensayo & Valor & Unidad \\
\hline \multirow[t]{3}{*}{1} & \multirow{3}{*}{$\begin{array}{l}\text { Adoquín } \\
\text { con un } 70 \% \\
\text { en reempla- } \\
\text { zo de agre- } \\
\text { gado fino }\end{array}$} & Tracción Indirecta & 3 & $\mathrm{MPa}$ \\
\hline & & $\begin{array}{l}\text { Resistencia a Compre- } \\
\text { sión }\end{array}$ & 31.62 & $\mathrm{MPa}$ \\
\hline & & $\begin{array}{l}\text { Absorción Total de } \\
\text { Agua }\end{array}$ & 8.83 & $\%$ \\
\hline \multirow[t]{3}{*}{2} & \multirow{3}{*}{$\begin{array}{l}\text { Adoquín } \\
\text { con un 50\% } \\
\text { en reempla- } \\
\text { zo de agre- } \\
\text { gado fino }\end{array}$} & Tracción Indirecta & 2.4 & $\mathrm{MPa}$ \\
\hline & & $\begin{array}{l}\text { Resistencia a Compre- } \\
\text { sión }\end{array}$ & 22.62 & $\mathrm{MPa}$ \\
\hline & & $\begin{array}{l}\text { Absorción Total de } \\
\text { Agua }\end{array}$ & 8.6 & $\%$ \\
\hline \multirow[t]{3}{*}{3} & \multirow{3}{*}{$\begin{array}{l}\text { Adoquín } \\
\text { con un 30\% } \\
\text { en reempla- } \\
\text { zo de agre- } \\
\text { gado fino }\end{array}$} & Tracción Indirecta & 2.7 & $\mathrm{MPa}$ \\
\hline & & $\begin{array}{l}\text { Resistencia a Compre- } \\
\text { sión }\end{array}$ & 27 & $\mathrm{MPa}$ \\
\hline & & $\begin{array}{l}\text { Absorción Total de } \\
\text { Agua }\end{array}$ & 7.83 & $\%$ \\
\hline
\end{tabular}

Tabla 5. Resultados de características mecánicas para diferentes dosificaciones de relave en reemplazo al agregado fino (polvo de piedra). Fuente: Departamento de Metalurgia Extractiva EPN, 2019.

Los resultados de tracción indirecta ejecutados en los adoquines fabricados con un $70 \%$ de relave en reemplazo del agregado fino, presentan una resistencia a la tracción de $3 \mathrm{MPa}$ y una resistencia a la compresión $31.62 \mathrm{MPa}$. Si bien este resultado no cumple con el valor mínimo promedio de resistencia a la tracción (3.6 $\mathrm{MPa}$ ) que exige la normativa INEN 3040, el resultado de resistencia a compresión simple (31.62 MPa) sobrepasa el valor requerido dentro de los establecido en los lineamientos de la normativa INEN 1488, la cual menciona que los adoquines elaborados para el 
uso de vías de tráfico peatonal y para estacionamientos deben cumplir el requerimiento de resistencia a la compresión de $20 \mathrm{MPa}$ y $30 \mathrm{MPa}$ respectivamente medidos a los 28 días.

Con lo expuesto en los acápites anteriores, en base a los resultados químicos, físicos y mineralógicos, resulta factible el uso de los relaves en la fabricación de adoquines para ser usados en beneficio de la comunidad o de las instalaciones del campamento de la empresa. Por otro lado, para lograr satisfactoriamente la fabricación de los adoquines se planea la siguiente metodología (Figura 3):

\section{Secado de piscina de relaves.}

2. Extracción y transporte del relave hacia el lugar de acopio.

3. Secado natural del relave para eliminar exceso de humedad.

4. Dosificación de los agregados en la mezcladora.

5. Vibro-prensado de adoquines.

6. Secado al aire libre por 28 días de los adoquines.
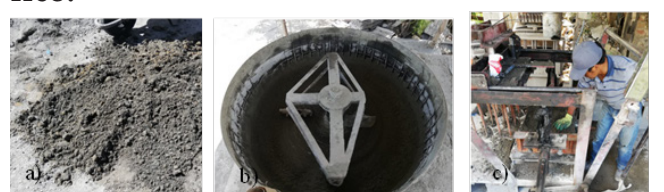

Figura 3. a) Secado natural del relave, b) Dosificación en la mezcladora, c) Vibro-prensado en moldes personalizados de adoquines, d) Secado al aire libre de los adoquines.

Uno de los objetivos de la empresa para el empleo de los relaves como agregado en la fabricación de adoquines, es usar estos elementos de apoyo en la mejora de las vías que son parte del campamento. Finalmente, en la Figura 4 se muestra la aplicación de los adoquines en la mejora en la red vial de tránsito peatonal en el interior del predio donde se desarrolla la mina. El área a intervenir con el uso de adoquines de relave es de $3311 \mathrm{~m} 2$, lo que equivale al evacuar una piscina de las 15 existentes en la empresa minera. En las siguientes figuras se observa la mejora que tendrían las vías.

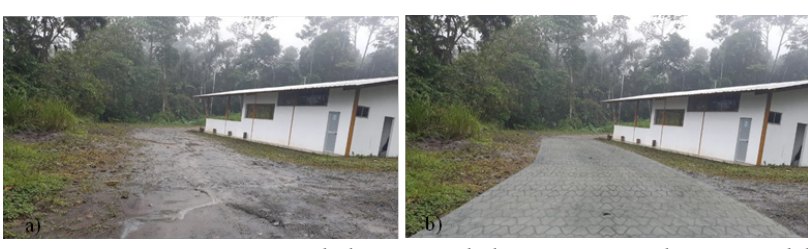

Figura 4. Vista panorámica de la mejora de las vías peatonales, sector del comedor. a) vía lastrada, b) vía con la implementación de adoquines de relave.

\section{CONCLUSIONES}

El uso de arenas de relave para la fabricación de adoquines fue factible en relación al cumplimiento de la norma INEN 3040 obteniendo como resultado una resistencia a la tracción indirecta de hasta $3 \mathrm{MPa}$, los prototipos pueden ser utilizados en zonas de tránsito peatonal y en zonas de estacionamiento. La dosificación para conformar a mezcla fue de 3/4: $1 \frac{1 / 2}{2}: 1: 2$ (cemento, agregado grueso, agregado fino, relave).

Mineralógicamente, el cuarzo es el mineral que predomina en los relaves, se presenta en porcentajes mayores al 75\% en todas las muestras y en base a los resultados de los adoquines prototipos, la presencia de este mineral ayudó a mejorar las propiedades físico mecánicas de los adoquines. Los relaves no muestran un potencial alto para generar drenaje ácido según los ensayos $\mathrm{ABA}$ (Acid-Base Accounting) realizados, por lo que no fue necesario un procedimiento adicional para eliminar elementos que pudieran causar un pasivo ambiental.

El aprovechamiento de los relaves con su uso en la fabricación de adoquines resulta factible en el ámbito económico, la fabricación de un adoquín alcanza un costo de USD 0.32. Como dato referencial la empresa al aprovechar el relave en la fabricación de adoquines ahorraría un aproximado de USD 95000 por la construcción de una relavera. En el contexto social la empresa generaría plazas de trabajo además de mitigar el pasivo ambiental por la generación de drenaje ácido (9). Desde el punto de vista técnico evitaría el invertir en estudios de prefactibilidad, factibilidad, construcción, cierre y monitoreo de las relaveras, con la puesta en marcha de un proyecto usando la metodología planteada en la investigación se llegaría una gestión óptima de los residuos mineros. 
eferencias

1. Pérez J. La mineria colonial americana bajo la dominacion española [Internet]. Universidad de las Palmas de Gran Canaria. Madrid; 2006. Available from: https://mdc.ulpgc.es/cgibin/showfile.exe?CISOROOT=/bolmc\&CISOPTR=130\&filename=131.pdf

2. Terram. La Minería y su Pasivo Ambiental. Análisis de Políticas Públicas [Internet].2003;24(12):116. Available from: https://www.terram.cl/descargar/recursos_naturales/mineria/app_analisis_de_politicas_publicas/APP-24-La-mineria-y-su-pasivo-ambiental.pdf

3. Dore E. Alternativas Latinoamericanas: Una interpretación socio-ecológica de la Historia minera Latinoamericana. Ecol política. 1994;79(7):49-68.

4. MINMINAS. Guía Ambiental Para el Manejo de Relaves Mineros. Ministerio de Minas y Energía. 2005;12:42. Available from: http://www.minem.gob.pe/minem/archivos/file/DGAAM/guias/relaveminero.pdf

5. González Sánchez Y, Fernández Díaz Y, Gutiérrez Soto T. El cambio climático y sus efectos en la salud. Rev Cubana Hig Epidemiol [Internet]. 2013;51(3):331-7. Available from: https://repositorio.cepal. org/bitstream/handle/11362/39855/S1501295_en.pdf?sequence $=1$

6. Vilela W, Espinsza M, Bravo A. La contaminación ambiental ocasionada por la minería en la provincia de El Oro. Rev Int Adm [Internet]. 2020;8(8):215-33. Available from: file://C:/Users/Pc/Downloads/2437-Artículo-7410-1-10-20201130.pdf

7. Soto R. Reaprovechamiento de relaves. Petroenergía [Internet]. 2019;07:1-32. Available from: https://www.petroenergia.info/revista-online-julio-2020

8. Romero AA, Medina R, Puente L, Flores SL, Guadalupe E, De E, et al. Los residuos sólidos mineros del proceso de flotación de minerales en la cuenca del rÍo Santa - Áncash. Rev del Inst Investig la Fac minas, Metal y ciencias geográficas. 2008;11(21):14-8.

9. Calderón JF. Desarrollo de adoquines, a partir de relaves de mina de la empresa minera "Agro-Industrial el Corazón" [Internet]. Universida Central del Ecuador; 2020. Available from: http://www.dspace. uce.edu.ec/handle/25000/21255

10. INAPI. Recuperación de metales valiosos desde residuos mineros [Internet]. Chile; 2021. Available from: https://www.inapi.cl/docs/default-source/2021/centro-de-documentacion/informes/informe-tecnologías-de-dominio-público/informe_dp_122.pdf?sfvrsn=e6ee5a40_0

11. Beltrán-rodríguez LN, Larrahondo JM, Cobos D. Emerging technologies for tailings disposal: opportunities for Colombian practice. Bol ciencias la Tierra [Internet]. 2018;44:5-20.

12. Silvana F, Romero A. Reuso de relaves mineros como insumo para la elaboración de agregados de construcción para fabricar ladrillos y baldosas. Ind Data [Internet]. 2010;13(1):75-82. Available from: redalyc.org/pdf/816/81619984010.pdf

13. Viveros MC. Aprovechamiento de relaves como agregados para la fabricación de adoquines - caso de estudio de relaves procedentes de la Planta de beneficio Campanillas. Quito-Ecuador; 2020.

14. Servicio Ecuatoriano de Normalización. NTE INEN 3040: Adoquines de hormigón. Requisitos y métodos de ensayo, Quito-Ecuador. 2016;45.

15. Servicio Ecuatoriano de Normalización. NTE INEN 1488: Adoquines. Requisitos, Quito -Ecuador. 1986;4.

16. Aduviri O. Tecnicas De Prevencion Y Control De La Generacion Acida En Mineria. Medio Ambient y Minería [Internet]. 2018;4(4):24-31. Available from: http://www.scielo.org.bo/scielo.php?script=sci_arttext\&pid=S2519-53522018000100003

17. White W, Lapakko K, Cox R. Static-test methods most commonly used to predict acid mine drainage: Practical Guidelines for Use and Interpretation. Environ Geochemistry Miner Depos Part A Theory Backgr. 2016;7A(June):325-38.

18. Yugsi A. Análisis de las propiedades mecánicas de adoquines elaborados con hormigón y polvillo de caucho de neumáticos reciclados y su correlación con adoquines convencionales [Internet]. Universidad Central del Ecuador; 2018.

19. Agency U. EP. Acid Mine Drainage Prediction [Internet]. 1994. p. 52. Available from: https:// 19january2017snapshot.epa.gov/sites/production/files/2015-09/documents/amd.pdf

20. Carles J, Joaquín A, De B, Geológica S, Melgarejo JC, Proenza JA, et al. Técnicas de caracteri- 
zación mineral y su aplicación en exploración y explotación minera. Bol Soc Geólogica Mex [Internet]. 2010;62:1-23. Available from: https://www.redalyc.org/pdf/943/94315961002.pdf

21. Huaman FE, Urbano G, Flores L. Caracterización preliminar de residuos de minería aluvial en la región Madre de Dios - Perú. Rev Cuba Química. 2020;32(2):1-13.

22. Cecioni AJ, Dick LA. Geologia del yacimiento epitermal de oro y plata can can, franja de maricunga, precordillera de copiapo, chile. Rev Geológica Chile [Internet]. 1992;19(1):3-17. Available from: https://app.ingemmet.gob.pe/biblioteca/pdf/RGC19-3.pdf

23. Romero AA, Flores SL, Medina R. Estudio de los metales pesados en el relave abandonado de Ticapampa. Rev del Inst Investig FIGMMG. 2008;11(11):13-6.

24. Morillo B, Santiago D, Privado I, Privado I. Análisis tenso deformacional de las obras de remediación implementadas para estabilizar la subsidencia minera bajo la Escuela La Inmaculada, Zaruma-Ecuador. FIGEMPA Investig y Desarro [Internet]. 2021;12:1-14. Available from: https://revistadigital.uce.edu. ec/index.php/RevFIG/article/view/3054/4262

25. Torres H, Patricio D, Cuasapaz G. La resistencia a la compresión y el desgaste en adoquines de concreto. Rev Arbitr Venez del Núcleo LUZ- Costa Orient del Lago. 2021;16(12):481-506. 